\title{
Diminish Electrostatic in Piezoresponse Force Microscopy through longer or ultra-stiff tips
}

\author{
A. Gomez ${ }^{a *}$, T. Puig ${ }^{a}$ and X. Obradors ${ }^{a}$ \\ anstitut de Ciència de Materials de Barcelona (ICMAB-CSIC), Campus UAB, Bellaterra, 08193, Catalonia, Spain \\ EMAIL: agomez@icmab.es
}

KeyWords: surface science, piezoresponse, atomic force microscopy, piezoelectricity, ferroelectricity, atomic force microscopy

\begin{abstract}
Piezoresponse Force Microscopy is a powerful but delicate nanoscale technique that measures the electromechanical response resulting from the application of a highly localized electric field. Though mechanical response is normally due to piezoelectricity, other physical phenomena, especially electrostatic interaction, can contribute to the signal read. We address this problematic through the use of longer ultra-stiff probes providing state of the art sensitivity, with the lowest electrostatic interaction and avoiding working in high frequency regime. In order to find this solution we develop a theoretical description addressing the effects of electrostatic contributions in the total cantilever vibration and its quantification for different setups. The theory is subsequently tested in a Periodically Poled Lithium Niobate (PPLN) crystal, a sample with well-defined 0 o and 180 ${ }^{\circ}$ domains, using different commercial available conductive tips. We employ the theoretical description to compare the electrostatic contribution effects into the total phase recorded. Through experimental data our description is corroborated for each of the tested commercially available probes. We propose that a larger probe length can be a solution to avoid electrostatic forces, so the cantilever-sample electrostatic interaction is reduced. Our proposed solution has great implications into avoiding artifacts while studying soft biological samples, multiferroic oxides, and thin film ferroelectric materials.
\end{abstract}

\section{Introduction}

Piezoresponse Force Microscopy (PFM) is a powerful tool based on Atomic Force Microscopy (AFM) to probe piezo and ferroelectric properties, at the nanoscale, in a wide variety of materials ranging from thin film complex oxides to biological samples[1-8]. In the PFM method, a conductive AFM tip is used in contact mode operation, while an AC Voltage signal is directly applied to the tip. As a result of the converse piezoelectric effect, the material vibrates at the same applied AC frequency. The vibration is recorded by the AFM photo-detector as a cantilever deflection signal, giving the desired piezoelectric properties characterization of the material[9-13].

Even though the method is simple, the interpretation of the resulting signal is not straightforward $[14,15]$. This situation is emphasized into two main problems: a ferroelectric like signal in non-ferroelectric materials[16] and the lack of quantitative results. Diging into the first problem, the scientific literature reports PFM images collected while studying nonferroelectric materials[17], as for example, recording a ferroelectric like behaviour in thin film 
cubic $\mathrm{HfO}_{2}$ or $\mathrm{SrTiO}_{3}[18-20]$. More importantly, the number of materials reporting ferroelectricity is growing accordingly to other authors[21]. For such reasons, solutions to avoid data misleading interpretation have been proposed[22]. Furthermore, the on-going solutions include methods to separate the electrostatic contribution from piezoelectricity [18], data mining[23], interferometer sensors as a replace to optical beam deflections systems[24], among others.

However, some challenges still remain, like diminishing the electrostatic forces from pure piezoelectric forces, which is crucial for studying thin film ferroelectric materials. Other authors have presented studies that include how such electrostatic signal will change the obtained results, from an experimental point of view[25-32]. In this work we tackle the problem through a new perspective: diminish electrostatic signal, rather than differentiating from the piezoelectricity. The effect of an undesired force proportional to the AC voltage is provided through solving Lock-in Amplifier (LIA) based theoretical models. The conclusions from this approach are tested experimentally in a well-known ferroelectric sample consisting of a Periodically Poled Lithium Niobate $\left(\mathrm{PPLiNbO}_{3}\right)$ [33]. The theory is used to improve the PFM signal through the study of different commercial available conductive tips, finding the tip that diminishes electrostatic contribution, while maintaining state of the art sensitivity. We find the lowest electrostatic influenced system through the use of longer tips, which are commercially available and usable in any AFM equipment.

\section{Theoretical Approach}

The electromechanical behaviour of the AFM tip in PFM experiments has been found to be related to different force interactions, being the most important ones piezoresponse and electrostatic. The electrostatic effect have been studied previously by other authors, demonstrating a ferroelectric-like signal in a non-ferroelectric material[14,34]. As electrostatic force is crucial, we develope a theoretical description to explain the effects of a small electrostatic signal in the overall signal. We assume two cantilever oscillations of the same frequency, with different amplitudes and different phases. One of these two signals corresponds to the vibration of the cantilever due to piezoelectric forces, while the other signal is the movement of the cantilever due to electrostatic interactions[35]. Both signals can be mathematically represented as a sum of individual signals:

$$
\mathbf{A}(\mathbf{t})=\mathbf{A}_{\text {Piezo }} \sin \left(\omega t+\emptyset_{\text {Piezo }}\right)+\mathbf{A}_{\text {Elec }} \sin \left(\omega t+\emptyset_{\text {Elec }}\right)
$$

Where $\mathbf{A}_{\text {Piezo }}, \emptyset_{\text {Piezo }}$ are the amplitude and phase of vibration due to piezoelectric forces; $\mathbf{A}_{\text {Elec }}, \emptyset_{\text {Elec }}$ are the Amplitude and Phase of vibration due to electrostatic forces and $w$ is the driving frequency of the $A C$ Voltage applied to the tip. We introduce both signals into the mathematical model of a LIA, to calculate the Phase of the whole signal, taking into account both piezoresponse and electrostatic interactions. The LIA uses the correlation function to obtain the amplitude and phase parameters of a given sinusoidal function[36]. We can use the correlation function with (1)

$$
R(\delta, T)=\frac{1}{T} \int_{0}^{T} f(t) A(t+\delta) d t(2)
$$


Where $T$ is the upper integration limit, $f(t)$ is the reference signal, $A(t)$ is the modulated response and $\delta$ is a time parameter. Using the integral properties we find:

$$
R(\delta, T)=\frac{1}{T} \int_{0}^{T} \sin (w t) A_{\text {Piezo }} \sin \left(w t+\emptyset_{\text {Piezo }}\right) d t+\frac{1}{T} \int_{0}^{T} \sin (w t) A_{\text {Elec }} \sin \left(w t+\emptyset_{\text {Elec }}\right) d t(3)
$$

The two independent integrals can be solved using standard Lock-in amplifiers theory[37]. Operating the integrals, it is found that the phase seen by the LIA has the following expression:

$$
\text { Phase }=\tan ^{-1}\left(\frac{\mathrm{U}_{1}}{\mathrm{U}_{2}}\right)(4)
$$

Where

$$
\begin{aligned}
\mathbf{U}_{1} & =\mathbf{A}_{\text {Piezo }} \cos \left(\emptyset_{\text {Piezo }}\right)+\mathbf{A}_{\text {Elec }} \cos \left(\emptyset_{\text {Elec }}\right)(5 a) \\
U_{2} & =A_{\text {Piezo }} \sin \left(\varnothing_{\text {Piezo }}\right)+A_{\text {Elec }} \sin \left(\emptyset_{\text {Elec }}\right)(5 b)
\end{aligned}
$$

the formula (4) is the expression of the phase for the global signal, including both piezoelectric and electrostatic interactions. According to the expression (4), it is shown that $\mathbf{A}_{\text {Elec }}$ is a parameter that can change the phase of the global signal. Even though electrostatic signal has been reported as a cause of data misleading[21] a full theoretical description has to be developed to understand the effects of this contribution to the global acquired signal $[17,18,21,38-44]$. To study the contribution of $A_{E l e c}$ into the global phase recorded by the LIA, we apply this theory to describe an ideal sample with up or down polarizations states. The electrostatic force is approximated as a constant phase force, because the DC bias is always 0 $\mathrm{V}[45,46]$. Mathematically, the conditions are depicted bellow:

$$
\emptyset_{\text {PiezoUP }}=0^{\mathrm{o}}, \emptyset_{\text {PiezoDOWN }}=180^{\circ}, \emptyset_{\text {Elec }}=30^{\circ}
$$

where $\emptyset_{\text {Piezoup }}, \emptyset_{\text {PiezoDown }}$ are the phase of the up and down polarization state and $\emptyset_{\text {Elec }}$ is the phase of the electrostatic forces, which will be equivalent to the phase value obtained in Electrostatic Force Microscopy (EFM) experiments. Due to the tip-sample work function difference, a phase of 30 was selected for modelling electrostatic signal phase. This value corresponds to an attractive force, commonly found when measuring electrostatic forces with an AFM. With the following conditions, we can study the resulting phase difference of domains up and down of the whole system as a function of the ratio between $A_{\text {Elec }} / A_{\text {Piezo }}$. In Figure 1, it is shown the phase of the up and down domains as well as the phase difference between both 


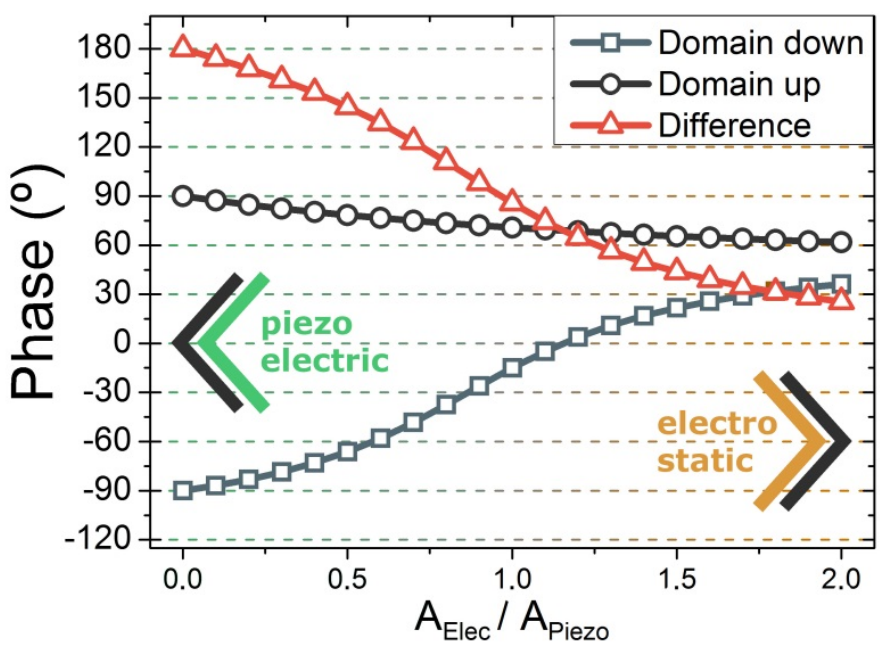

Figure 1. Theoretical representation of the total phase difference versus $A_{\text {Elec }} / A_{\text {Piezo }}$ for a sample with antiparallel domains. If no electrostatic forces are involved, the phase difference between antiparallel domains is $180^{\circ}$. As soon as electrostatic contribution appears, the total phase of the system decreases with the increased $A_{E l e c} / A_{\text {Piezo }}$ ratio.

domains, versus the relation $A_{\text {Elec }} / A_{\text {Piezo }}$. As the $A_{\text {Elec }} / A_{\text {Piezo }}$ ratio increases, the phase difference between up and down polarization states decreases. Moreover, the phase change between up and down domains is not symmetric. This asymmetric contribution is related to the difference between the electrostatic signal phase and the piezoresponse phase for each of the domains. The electrostatic phase, 30 , is closer to the up domain phase, which is 0 ㅇ, and hence its contribution is lowered compared to the down domain case. In order to further elucidate the influence of the $\emptyset_{\text {Elec }}$ employed we replicate the graphs for different phase values obtaining the same trend (see S1 in Supplementary Information).

Here we select three representative situations to emphasize the relevance of the $A_{\text {Elec }} / A_{\text {Piezo }}$ ratio. For high electrostatic contribution, $A_{\text {Elec }} / A_{\text {Piezo }}$ equals to 2 or greater, the domains cannot be seen and, moreover, the global phase of each domain is proportional to the electrostatic phase signal, see Figure 2. 


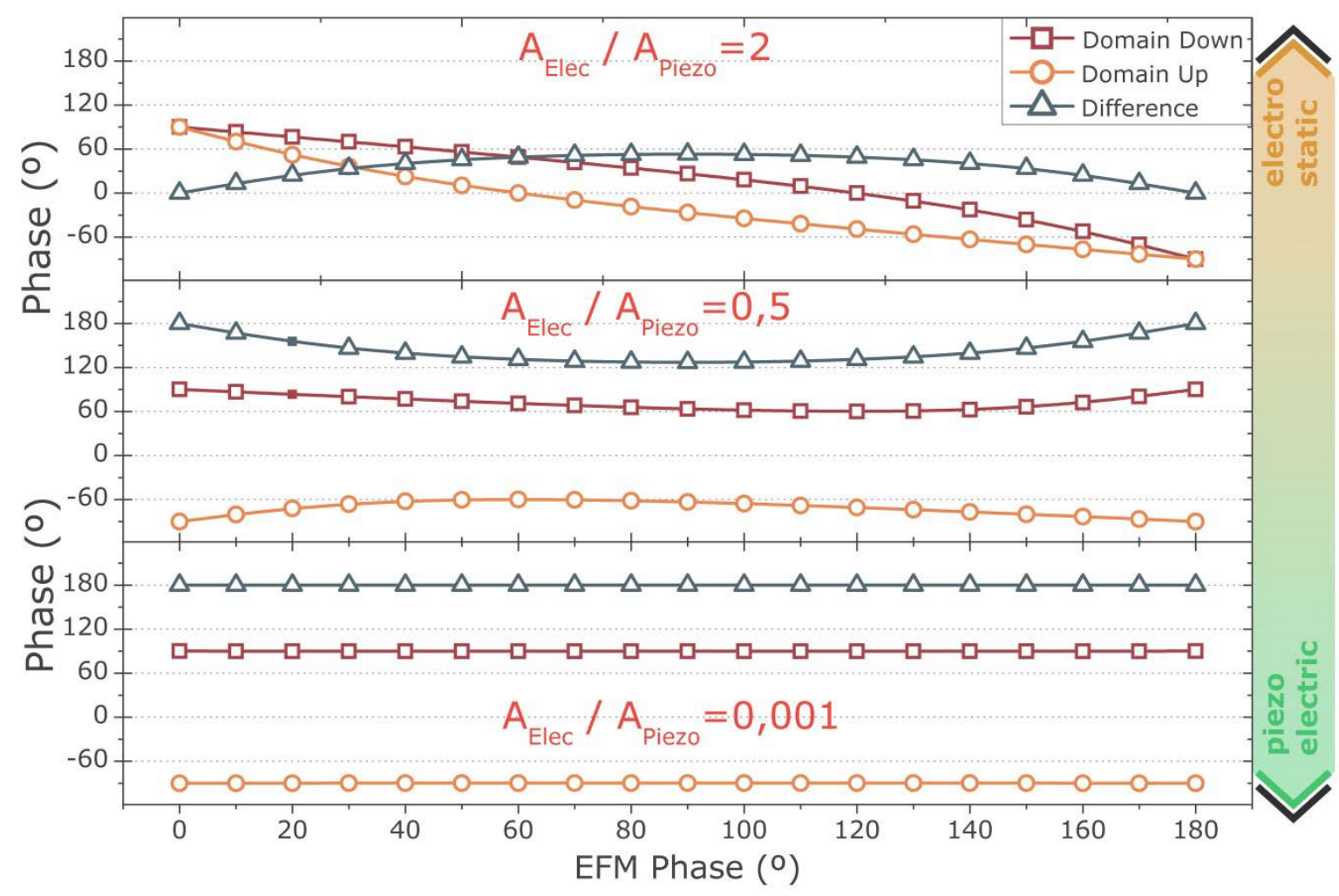

Figure 2. Theoretical description of the total phase difference versus the electrostatic signal phase, for different ratios of $A_{\text {Elec }} / A_{\text {Piezo }}$. For the case where the ratio is 0.001 , the phase difference read is independent of the EFM phase signal. If this ratio increases, the phase for each of the domains decreases, up to a point that all the phase recorded is directly proportional to the EFM phase signal and independent of the piezoelectric signal.

As a consequence, a change of the electrostatic phase signal dominates the global phase recorded, which is independent of the piezoresponse signal phase. In this region, a ferroelectric like response in a non-ferroelectric material can be obtained. For the low electrostatic contribution regime, $A_{\text {Elec }} / A_{\text {Piezo }}$ is 0.001 , the domains have a homogeneous 180 - phase difference; the majority of the signal comes from the piezoresponse signal. For $A_{\text {Elec }} / A_{\text {Piezo }}=0.5$, the global phase measured is 1260 , diminishing the phase difference being read between the two states. Other effects of electrostatic contribution, regarding the spatial resolution and width of domain walls recorded in PFM cannot be evaluated with our model, even though there are other suitable models for such purpose[25,31,32,47]. Yet the model gives a direct and easy experimental view of the effect of electrostatic signal, it cannot be directly implemented in other samples, as we should have into account other experimental related issues like a background signal, ionic mobility, trapped charge, among others[27$30,48]$. 


\section{Experimental Results}

At this step, we employed the described theory to experimentally illustrate the relevance of $\mathrm{A}_{\text {Elec }} / \mathrm{A}_{\text {Piezo }}$. We studied a PPLN crystal, which presents well-defined 0 o and 180 o polarization states, using different commercial available conductive tips (see Table 1).

Table 1: Specifications comparison between different conductive tips available in the market

\begin{tabular}{|c|c|c|c|c|c|c|}
\hline Tip name & $\mathrm{k}(\mathrm{N} / \mathrm{m})$ & $\begin{array}{c}\text { Sensitivity } \\
(\mathrm{nm} / \mathrm{V})\end{array}$ & $\begin{array}{c}\text { Cantilever } \\
\text { Length }(\mu \mathrm{m})\end{array}$ & $\begin{array}{c}\text { Resonance } \\
\text { Frequency(kHz) }\end{array}$ & $\begin{array}{c}\text { Tip } \\
\text { Material }\end{array}$ & Tip Length $(\mu \mathrm{m})$ \\
\hline $\begin{array}{c}\text { NanoWorld EFM (Platinum) } \\
\text { NanoWorld CDT-FMR } \\
\text { (Diamond) }\end{array}$ & 2.8 & 120.5 & 225 & 75 & 15 \\
\hline $\begin{array}{c}\text { AppNano DDSICONA (Soft } \\
\text { diamond) }\end{array}$ & 0.2 & 257.1 & 225 & 105 & Diamond & 15 \\
\hline $\begin{array}{c}\text { RockyM 25PT400 (Longer) } \\
\text { RockyM 25PT300 }\end{array}$ & 8 & 384.6 & 400 & 12 & Diamond & 15 \\
\hline $\begin{array}{c}\text { RockyM 25PT200B-H (Ultra- } \\
\text { stiff) }\end{array}$ & 250 & 248.2 & 200 & 300 & Solid Pt & 80 \\
\hline
\end{tabular}

Table 1. Comparison between the different conductive tips used to carry on PFM measurement which are commercially available.

For PFM measurements, a general consensus is to use a stiff cantilever to reduce electrostatic signal[20]. A stiff cantilever has a lower thermal noise, while a stiffer cantilever will drop its sensitivity and increase the contact resonance frequency to the Megahertz range[49]. However, other tip parameters have not been considered in order to reduce the electrostatic influence. In the case of Rocky Mountain Nanotechnology (RMN) tips the larger tip length may play an important role into diminish electrostatic signal (see S2 in Supplementary Information). Such a large tip length greatly reduces the cantilever-sample capacitive coupling and therefore the electrostatic contribution. Larger tip length probes have been custom manufactured and employed for imaging biological samples, but the use of a larger tip length to avoid electrostatic interactions is a solution which was not implemented, tested and demonstrated previously[50]. Another advantage of using RMN tips is the aspect ratio of the tip, as being sharper, the capacitive coupling between the tip and the sample is also reduced[51]. At this point, the test sample is scanned with three conductive tips, all of them commercially available, which are NanoWorld EFM (NW EFM), RockyMountain Nanotechnology 25PT400 
(RMN-25PT400) and RockyMountain Nanotechnology 25PT200H (RMN-25PT200H-B). We specifically selected these probes as the first one is a platinum coated tip, which is the most common in PFM experiments, while the second is a larger tip length probe, with equivalent spring constant. The third probe, RMN-25PT200H-B consists of an ultra-stiff probe, with spring constant of $250 \mathrm{~N} / \mathrm{m}$, maintaining good deflection sensitivity and a contact resonance frequency of $240 \mathrm{kHz}$.

We scan a $10 \times 10$ microns area, located in the same spot of the sample, to minimize possible sample in-homogeneities. A similar force is applied between tip and sample to exclude the force as a variable, unless for the case of the Ultra-stiff tips, where it is not possible to match the exact force employed as for the other tips [15]. The scanned area is selected between two opposite domains of the sample. The AC voltage frequency is kept constant at $105 \mathrm{kHz}$. Such frequency is chosen as it is far from the contact resonance frequency of any of the three tips, avoiding resonant artefacts. We start the image applying $5 \mathrm{VAC}$, and we increase the AC voltage amplitude in $1 \mathrm{~V}$ steps, each 2 microns, until a maximum of 9 VAC is achieved, see Figure 3.

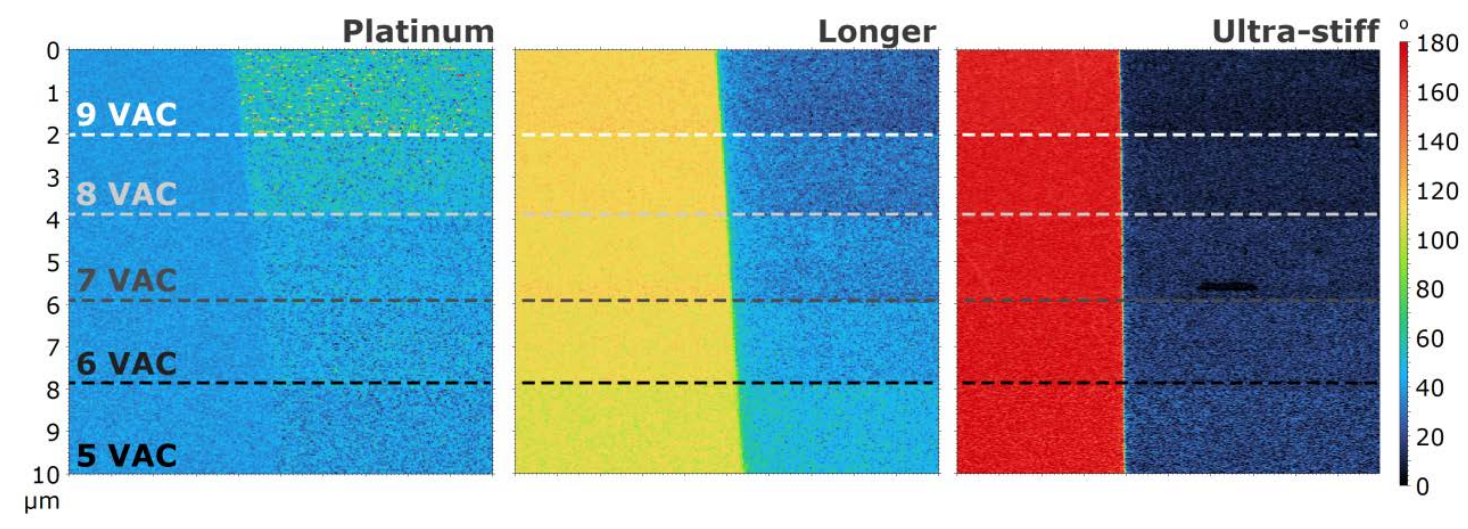

Figure. 3. From left to right, $10 \times 10 \mu \mathrm{m}$ PFM Phase images of two antiparallel domains of a PPLN, obtained with a platinum coated tip (NW EFM), a longer solid platinum tip (RMN25PT400) and ultra-stiff tip (RMN-25PT200H). $105 \mathrm{kHz}$ AC voltage was applied to the tip, the amplitude was increased from $5 \mathrm{VAC}$ to $9 \mathrm{VAC}$, the amplitude was increased at a pace of $1 \mathrm{~V}$ each 2 microns-bottom to top.

As electrostatic forces tend to saturate at higher bias, we can assume that from 5VAC to 9VAC electrostatic force is maintained constant[52]. With this assumption, we can interpret the contrast change in the image as an increase in the piezoelectric signal over the electrostatic signal, each time the applied AC bias increases. It is observed that by increasing the AC bias, the phase difference between the up and down domains is also increased (see S3 in SI). The proposed methodology was tested using two different tips, with the exact same part number, in order to test the reproducibility of the results (see S4 in SI). Furthermore, the increased phase is not the same for up and down domain phases, an asymmetry is found (see S5 in SI). This asymmetry was not previously explained; however the proposed theoretical description 
explains this experimental result, as the electrostatic signal interacts greater with the down domain phase, compared to the up domain. Other authors have shown a background signal related to the instrumentation phase offset present in lock-in amplifiers and associated electronic instrument, which are inherent to the equipment used but not to the specific measurement conditions[26].The amplitude recorded along the three phase images is further included (see S6 in SI).

Through the experimental data, we can compare which of the setups provides a piezoelectric signal less influenced by electrostatic forces. The ideal case consists of reading a full 180은 phase signal between domains, independently of the AC voltage used. The experimental data was fitted in the theoretical values of Figure 1, where fitting is shown in Figure 4 for a $\emptyset_{\text {Elec }}$ of

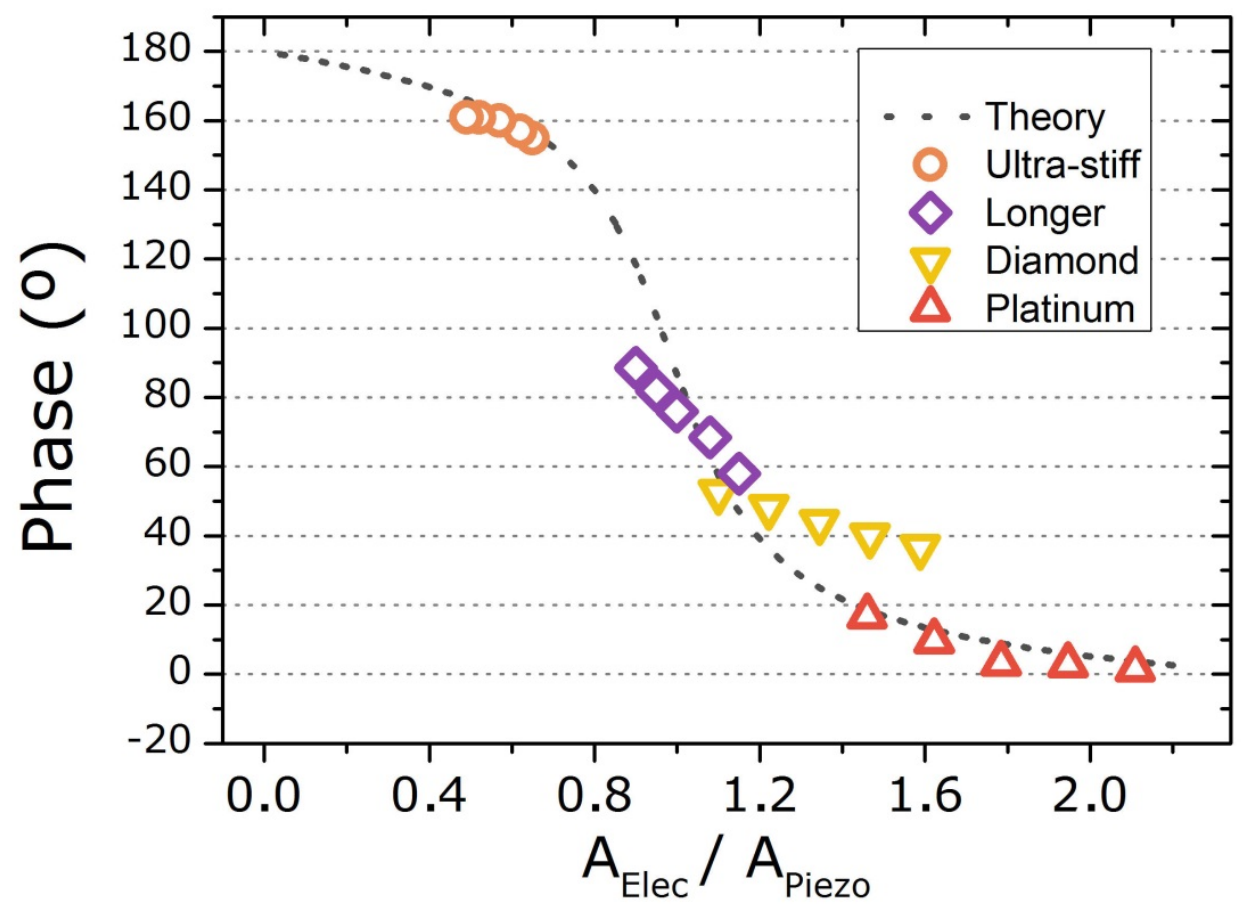

Figure 4. Phase difference between up and down domains versus $A_{\text {Elec }} / A_{\text {Piezo }}$ ratio. Dotted line corresponds to the theoretical prediction while experimental data is fitted into the curve, corresponding to the ultra-stiff, longer, diamond and platinum coated tips. It is seen that the less electrostatically influenced tip is the ultra-stiff, followed by longer tips.

9o. Within these conditions, we find that the electrostatic contribution of the longer tip is 1.9 times lower than standard platinum tip and 1.5 times lower than a diamond coated tip (NW CDT-FMR)(see S7 of SI). For ultra-stiff tips, we find that the electrostatic contribution is 2.9 times lower compared to standard platinum tip and 2.03 compared to a diamond coated tip. Even though the electrostatic contribution cannot be fully separated from piezoresponse signal, its contribution can be greatly diminished by optimizing the setup used. 
Finally, we performed another experiment to corroborate our previous findings. We change the phase of the electrostatic signal phase by applying a DC bias to the sample. We use a diamond doped DD-SICONA probe, with very low $k$ constant cantilever of $0.2 \mathrm{~N} / \mathrm{m}$, which is extremely sensitive to little forces as Electrostatic ones, as well as cantilever buckling[15]. PFM phase image, Figure 5a, of the test sample, were acquired with an AC Voltage of $5 \mathrm{~V}$ amplitude while applying a $D C$ bias of $+5 \mathrm{~V}$ from its bottom to the middle part and a DC bias of $-5 \mathrm{VDC}$ from the middle to the upper part.
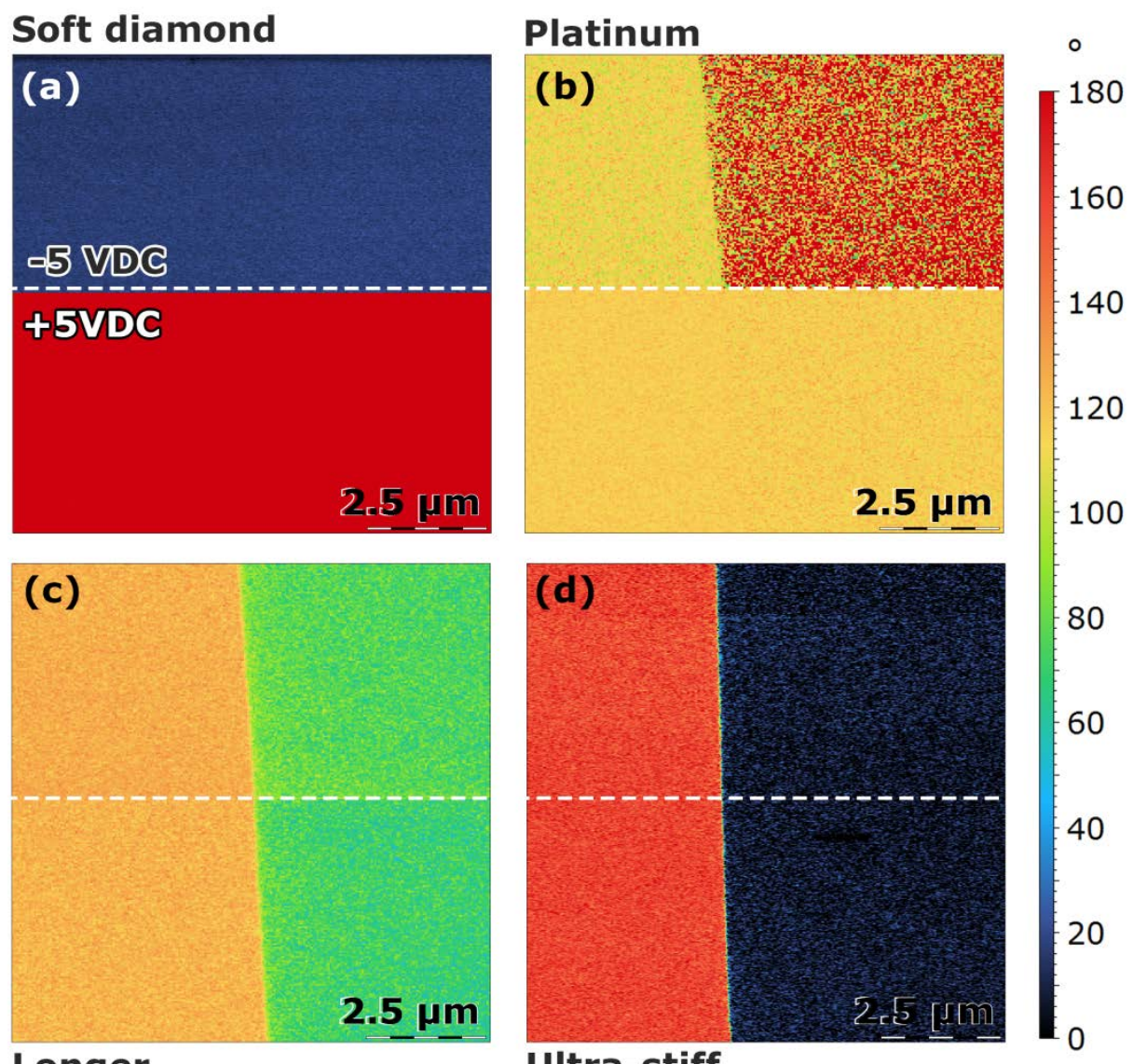

Longer

Ultra-stiff

Figure 5. PFM phase image obtained with a soft diamond coated probe (a), a standard platinum coated tip (b), a longer tip length probe (c) and a ultra-stiff probe (d). Image acquired with 5 Volts AC voltage, $105 \mathrm{kHz}$ frequency, $+5 \mathrm{VDC}$ was applied to the sample from bottom to the middle, while -5VDC is applied from middle to bottom. It is found that both soft diamond and platinum tip are extremely sensitive to DC bias, while longer ultra-stiff tips are independent of such applied DC bias.

As denoted by PFM images, (see S8 of SI) the ferroelectric domains are vertically aligned, however the piezoelectric signal is completely overlapped by electrostatic contribution. We 
can use our theory to explain this result, as we are now in the regime where $A_{\text {Elec }} / A_{\text {Piezo }}$ is greater than 2, where a change of $180 \circ$ of $\emptyset_{\text {Elec }}$ shifts the global phase of the system by 180․ This phase shift is not related piezoelectricity, as we are far away from the coercive field of the crystal[ $[40,53]$. The same experiment was repeated with other three tips, a standard platinum coated tip, Figure $\mathbf{5 b}$, a longer tip, Figure $\mathbf{5 c}$ and an ultra-stiff tip, Figure $\mathbf{5 d}$. It is found that the less influenced by electrostatic properties is the ultra-stiff tip, while the longer tip length probe provides a less electrostatically influenced signal, compared with its counterpart, the standard platinum coated tip. Through this experiment, we can confirm our proposed solution to diminish the electrostatic contribution by classifying the tips as a function of $A_{\text {Elec }} / A_{\text {Piezo }}$ ratio.

\section{Conclusions}

We propose a theoretical description to compare the effect of electrostatic forces in Piezoresponse Force Microscopy (PFM) for different setups. By employing a mathematical description of the lockin amplifier, we theoretically demonstrate the effect of a electrostatic signal into the PFM signal. The theoretical description is subsequently validated through a an experimental methodology in order to quantify the electrostatic contribution for different case scenarios. The method, based in scanning a Periodically Polled Lithium Niobate $\left(\mathrm{PPLiNbO}_{3}\right)$ is presented in order to quantify and compare the electrostatic contribution of different conductive tips available in the market. The proposed method does not need any special equipment or modified setup. After studying commercially available conductive tips for PFM, we compare the electrostatic influence for each tip, finding the one which minimizes the electrostatic contribution, maximizes deflection sensitivity value and which resonates below the $\mathrm{MHz}$ range, achieving state of the art PFM measurements. Our proposed solution, using longer or longer and ultra-stiff tips can be immediately implemented in any AFM setup without any physical modification.

\section{Experimental setup}

The test sample is a Periodically Poled Lithium Niobate (PPLN) consisting of antiparallel domains, with a domain size of 10-50 $\mu \mathrm{m}$ width and $400 \mu \mathrm{m}$ length. The sample, which is commercially available, has a piezoelectric coefficient $d_{33}$ of $7.5 \mathrm{pm} / \mathrm{V}$, coercive field $2 \times 10^{7} \mathrm{~V} / \mathrm{m}$ and surface polarization of $0.7 \mathrm{C} / \mathrm{m}^{2}$ [53]. The equipment used is an Agilent 5500 SPM with the AC Mode III accessory. The AC frequency is $105 \mathrm{kHz}$ for all PFM measurements, out of resonance, to avoid possible resonant artefacts. All the images were acquired in low ambient humidity conditions, less than $8 \%$.

\section{Supplementary Material}

See supplementary material for the complete description of each supplementary information experiments.

\section{Acknowledgements}

This research was supported by Consolider NANOSELECT (CSD 2007-00041). Supported by the Spanish Ministry of Economy and Competitiveness (MINECO, MAT2014-51778-C2-1-R project, co-financed with FEDER). ICMAB acknowledges financial support from the Spanish Ministry of 
Economy and Competitiveness, through the "Severo Ochoa" Programme for Centres of Excellence in R\&D (SEV- 2015-0496). The authors thank ICMAB Scientific and Technical Services. M.C acknowledges RyC contract 2013-12448.

\section{References}

[1] F. Felten, G.A. Schneider, J.M. Saldana, S. V Kalinin, J.M. Saldaña, S. V Kalinin, Modeling and measurement of surface displacements in $\mathrm{BaTiO} 3$ bulk material in piezoresponse force microscopy, J. Appl. Phys. 96 (2004) 563-568. doi:10.1063/1.1758316.

[2] M.H. Zhao, Z.L. Wang, S.X. Mao, Piezoelectric characterization individual zinc oxide nanobelt probed by piezoresponse force microscope, Nano Lett. 4 (2004) 587-590. doi:10.1021/nl035198a.

[3] Z.L. Wang, Energy harvesting for self-powered nanosystems, Nano Res. 1 (2008) 1-8. doi:10.1007/s12274-008-8003-x.

[4] S. Xu, Z.L. Wang, One-dimensional $\mathrm{ZnO}$ nanostructures: Solution growth and functional properties, Nano Res. 4 (2011) 1013-1098. doi:10.1007/s12274-011-0160-7.

[5] H.D. Espinosa, R.A. Bernal, M. Minary-Jolandan, A review of mechanical and electromechanical properties of piezoelectric nanowires, Adv. Mater. 24 (2012) 46564675. doi:10.1002/adma.201104810.

[6] Y. Liu, Y. Zhang, M.J. Chow, Q.N. Chen, J. Li, Biological ferroelectricity uncovered in aortic walls by piezoresponse force microscopy, Phys. Rev. Lett. 108 (2012) 078103. doi:10.1103/PhysRevLett.108.078103.

[7] C.B. Pierre, D.G. Coll, Soft-Chemistry-Based Routes to Epitaxial - Quartz Thin Films with Tunable Textures, 340 (2013) 827-832. doi:10.1126/science.1232968.

[8] A. Queralt??, A. P??rez Del Pino, M. De La Mata, J. Arbiol, X. Obradors, T. Puig, Ultrafast crystallization of Ce0.9Zr0.102-y epitaxial films on flexible technical substrates by pulsed laser irradiation of chemical solution derived precursor layers, Cryst. Growth Des. 15 (2015) 1957-1967. doi:10.1021/acs.cgd.5b00115.

[9] M. Alexe, A. Gruverman, Nanoscale Characterisation of Ferroelectric Materials, Springer, 2004. doi:10.1007/978-3-662-08901-9.

[10] O. Kolosov, Kolosov Replies:, Phys. Rev. Lett. 76 (1996) 4292-4292. doi:10.1103/PhysRevLett.76.4292.

[11] S. V. Kalinin, a. Rar, S. Jesse, A decade of piezoresponse force microscopy: progress, challenges, and opportunities, IEEE Trans. Ultrason. Ferroelectr. Freq. Control. 53 (2006) 2226-2252. http://ieeexplore.ieee.org/xpls/abs_all.jsp?arnumber=4037257.

[12] S. V Kalinin, A.N. Morozovska, L.Q. Chen, B.J. Rodriguez, Local polarization dynamics in ferroelectric materials, Reports Prog. Phys. 73 (2010) 056502. doi:10.1088/00344885/73/5/056502.

[13] A. Gómez, J.M. Vila-Fungueiriño, R. Moalla, G. Saint-Girons, J. Gázquez, M. Varela, R. 
Bachelet, M. Gich, F. Rivadulla, A. Carretero-Genevrier, Electric and Mechanical Switching of Ferroelectric and Resistive States in Semiconducting BaTiO3-סFilms on Silicon, Small. 13 (2017). doi:10.1002/smll.201701614.

[14] D. a Bonnell, D. a. Bonnell, S. V Kalinin, S.V. Kalinin, a L. Kholkin, a. L. Kholkin, a Gruverman, a. Gruverman, Piezoresponse Force Microscopy: A Window into Electromechanical Behavior at the Nanoscale, MRS Bull. 34 (2009) 648-657. doi:10.1557/mrs2009.176.

[15] S. Kalinin, D. Bonnell, Imaging mechanism of piezoresponse force microscopy of ferroelectric surfaces, Phys. Rev. B. 65 (2002) 1-11. doi:10.1103/PhysRevB.65.125408.

[16] R.K. Vasudevan, N. Balke, P. Maksymovych, S. Jesse, S. V. Kalinin, Ferroelectric or nonferroelectric: Why so many materials exhibit "ferroelectricity" on the nanoscale, Appl. Phys. Rev. 4 (2017). doi:10.1063/1.4979015.

[17] N. Balke, P. Maksymovych, S. Jesse, I.I. Kravchenko, Q. Li, S. V Kalinin, Exploring Local Electrostatic E ff ects with Scanning Probe Microscopy : Implications for Piezoresponse Force Microscopy and Triboelectricity, (2014) 10229-10236.

[18] N. Balke, P. Maksymovych, S. Jesse, A. Herklotz, A. Tselev, C.B. Eom, I.I. Kravchenko, P. $\mathrm{Yu}, \mathrm{S}$. V. Kalinin, Differentiating Ferroelectric and Nonferroelectric Electromechanical Effects with Scanning Probe Microscopy, ACS Nano. 9 (2015) 6484-6492. doi:10.1021/acsnano.5b02227.

[19] J. Zhang, C.Y. Wang, C. Bowen, Piezoelectric effects and electromechanical theories at the nanoscale, Nanoscale. 6 (2014) 13314-13327. doi:10.1039/c4nr03756a.

[20] M. Andrä, F. Gunkel, C. Bäumer, C. Xu, R. Dittmann, R. Waser, The influence of the local oxygen vacancy concentration on the piezoresponse of strontium titanate thin films., Nanoscale. 7 (2015) 14351-7. doi:10.1039/c5nr03643g.

[21] H. Miao, C. Tan, X. Zhou, X. Wei, F. Li, More ferroelectrics discovered by switching spectroscopy piezoresponse force microscopy?, EPL (Europhysics Lett. 108 (2014) 27010. doi:10.1209/0295-5075/108/27010.

[22] S. Jesse, A.P. Baddorf, S. V. Kalinin, Switching spectroscopy piezoresponse force microscopy of ferroelectric materials, Appl. Phys. Lett. 88 (2006) 21-24. doi:10.1063/1.2172216.

[23] S. Jesse, B.J. Rodriguez, S. Choudhury, A.P. Baddorf, I. Vrejoiu, D. Hesse, M. Alexe, E. a Eliseev, A.N. Morozovska, J. Zhang, L.-Q. Chen, S. V Kalinin, Direct imaging of the spatial and energy distribution of nucleation centres in ferroelectric materials., Nat. Mater. 7 (2008) 209-215. doi:10.1038/nmat2114.

[24] A. Labuda, R. Proksch, Quantitative measurements of electromechanical response with a combined optical beam and interferometric atomic force microscope, Appl. Phys. Lett. 106 (2015) 253103. doi:10.1063/1.4922210.

[25] E.A. Eliseev, S. V. Kalinin, S. Jesse, S.L. Bravina, A.N. Morozovska, Electromechanical detection in scanning probe microscopy: Tip models and materials contrast, J. Appl. Phys. 102 (2007). doi:10.1063/1.2749463.

[26] T. Jungk, A'. Hoffmann, E. Soergel, Consequences of the background in piezoresponse force microscopy on the imaging of ferroelectric domain structures, J. Microsc. 227 
(2007) 72-78. doi:10.1111/j.1365-2818.2007.01783.x.

[27] S. V. Kalinin, S. Jesse, B.J. Rodriguez, E.A. Eliseev, V. Gopalan, A.N. Morozovska, Quantitative determination of tip parameters in piezoresponse force microscopy, Appl. Phys. Lett. 90 (2007) 1-4. doi:10.1063/1.2742900.

[28] S. V. Kalinin, S. Jesse, B.J. Rodriguez, K. Seal, A.P. Baddorf, T. Zhao, Y.H. Chu, R. Ramesh, E.A. Eliseev, A.N. Morozovska, B. Mirman, E. Karapetian, Recent advances in electromechanical imaging on the nanometer scale: Polarization dynamics in ferroelectrics, biopolymers, and liquid imaging, Japanese J. Appl. Physics, Part 1 Regul. Pap. Short Notes Rev. Pap. 46 (2007) 5674-5685. doi:10.1143/JJAP.46.5674.

[29] S. V. Kalinin, B.J. Rodriguez, S. Jesse, E. Karapetian, B. Mirman, E. a. Eliseev, A.N. Morozovska, Nanoscale Electromechanics of Ferroelectric and Biological Systems: A New Dimension in Scanning Probe Microscopy, Annu. Rev. Mater. Res. 37 (2007) 189238. doi:10.1146/annurev.matsci.37.052506.084323.

[30] A.N. Morozovska, E.A. Eliseev, G.S. Svechnikov, V. Gopalan, S. V. Kalinin, Effect of the intrinsic width on the piezoelectric force microscopy of a single ferroelectric domain wall, J. Appl. Phys. 103 (2008). doi:10.1063/1.2939369.

[31] A.N. Morozovska, E.A. Eliseev, S.L. Bravina, S. V Kalinin, Resolution-function theory in piezoresponse force microscopy: Wall imaging, spectroscopy, and lateral resolution, Phys. Rev. B - Condens. Matter Mater. Phys. 75 (2007) 1-18. doi:10.1103/PhysRevB.75.174109.

[32] A.N. Morozovska, S. V Svechnikov, E.A. Eliseev, S. V Kalinin, Extrinsic size effect in piezoresponse force microscopy of thin films, Phys. Rev. B - Condens. Matter Mater. Phys. 76 (2007) 1-5. doi:10.1103/PhysRevB.76.054123.

[33] S. Wu, Piezoresponse force microscopy, Phys. Rev. Lett. 100 (2008) 097601. doi:10.1088/0022-3727/44/46/464003.

[34] R. Proksch, In-situ piezoresponse force microscopy cantilever mode shape profiling, J. Appl. Phys. 118 (2015). doi:10.1063/1.4927809.

[35] U. Rabe, K. Janser, W. Arnold, Vibrations of free and surface-coupled atomic force microscope cantilevers: Theory and experiment, Rev. Sci. Instrum. 67 (1996) 3281. doi:10.1063/1.1147409.

[36] L.A. Waistein, V.D. Zubakov, Extraction of signals from noise, Dover Publications Inc., 1970. doi:10.1119/1.1969250.

[37] T.H. Wilmshurst, Signal Recovery from Noise in Electronic Instrumentation, Bristol, 1990. doi:10.1049/ep.1985.0494.

[38] J. Hong, K. Noh, S. Park, S. Kwun, Z. Khim, Surface charge density and evolution of domain structure in triglycine sulfate determined by electrostatic-force microscopy, Phys. Rev. B. 58 (1998) 5078-5084. doi:10.1103/PhysRevB.58.5078.

[39] M. Honig, J.A. Sulpizio, J. Drori, A. Joshua, E. Zeldov, S. Ilani, Local electrostatic imaging of striped domain order in LaAlO3/SrTiO3., Nat. Mater. 12 (2013) 1112-1118. doi:10.1038/nmat3810.

[40] U. Bostanci, M.K. Abak, O. Aktas, A. Dâna, Nanoscale charging hysteresis measurement 
by multifrequency electrostatic force spectroscopy, Appl. Phys. Lett. 92 (2008) 093108. doi:10.1063/1.2888765.

[41] E.A. Eliseev, A.N. Morozovska, A. V. levlev, N. Balke, P. Maksymovych, A. Tselev, S. V. Kalinin, Electrostrictive and electrostatic responses in contact mode voltage modulated scanning probe microscopies, Appl. Phys. Lett. 104 (2014) 6-11.

doi:10.1063/1.4882861.

[42] S. Lepadatu, M. Stewart, M.G. Cain, Quantification of electromechanical coupling measured with piezoresponse force microscopy, J. Appl. Phys. 116 (2014) 066806. doi:10.1063/1.4891353.

[43] P. Zubko, N. Jecklin, A. Torres-Pardo, P. Aguado-Puente, A. Gloter, C. Lichtensteiger, J. Junquera, O. Stéphan, J.M. Triscone, Electrostatic coupling and local structural distortions at interfaces in ferroelectric/paraelectric superlattices, Nano Lett. 12 (2012) 2846-2851. doi:10.1021/nl3003717.

[44] M. Riba-Moliner, N. Avarvari, D.B. Amabilino, A. González-Campo, A. Gómez, Distinguishing between Mechanical and Electrostatic Interaction in Single Pass Multi Frequency Electrostatic Force Microscopy Measurements on a Molecular Material, Langmuir. 32 (2016) 13593-13599. doi:10.1021/acs.langmuir.6b03390.

[45] I.H. Campbell, J.D. Kress, R.L. Martin, D.L. Smith, N.N. Barashkov, J.P. Ferraris, Controlling charge injection in organic electronic devices using self-assembled monolayers, Appl. Phys. Lett. 71 (1997) 3528. doi:10.1063/1.120381.

[46] A. Kumar, T.M. Arruda, A. Tselev, I.N. Ivanov, J.S. Lawton, T. a Zawodzinski, O. Butyaev, S. Zayats, S. Jesse, S. V Kalinin, Nanometer-scale mapping of irreversible electrochemical nucleation processes on solid Li-ion electrolytes., Sci. Rep. 3 (2013) 1621. doi:10.1038/srep01621.

[47] S. Physics, N. Academy, To be submitted to Phys. Rev. B, Sci. Technol. (n.d.) 1-76.

[48] S. Santos, C.-Y. Lai, T. Olukan, M. Chiesa, Multifrequency AFM: from origins to convergence, Nanoscale. 9 (2017) 5038-5043. doi:10.1039/C7NR00993C.

[49] H.J. Butt, M. Jaschke, Calculation of thermal noise in atomic force microscopy, Nanotechnology. 6 (1995) 1. doi:10.1088/0957-4484/6/1/001.

[50] M. Shibata, T. Uchihashi, T. Ando, R. Yasuda, Long-tip high-speed atomic force microscopy for nanometer-scale imaging in live cells, Sci. Rep. 5 (2015) 8724. doi:10.1038/srep08724.

[51] J.W. Hong, S. Park, Z.G. Khim, Measurement of hardness, surface potential, and charge distribution with dynamic contact mode electrostatic force microscope, Rev. Sci. Instrum. 70 (1999) 1735. doi:10.1063/1.1149660.

[52] D. Seol, S. Park, O. V. Varenyk, S. Lee, H.N. Lee, A.N. Morozovska, Y. Kim, Determination of ferroelectric contributions to electromechanical response by frequency dependent piezoresponse force microscopy, Sci. Rep. 6 (2016). doi:10.1038/srep30579.

[53] R.S. Weis, T.K. Gaylord, Lithium niobate: Summary of physical properties and crystal structure, Appl. Phys. A Solids Surfaces. 37 (1985) 191-203. doi:10.1007/BF00614817. 